\title{
Educational and behavioural interventions for anticoagulant therapy in patients with atrial fibrillation (Protocol)
}

\author{
Smith DE, Borg Xuereb C, Pattison HM, Lip GYH, Lane DA
}

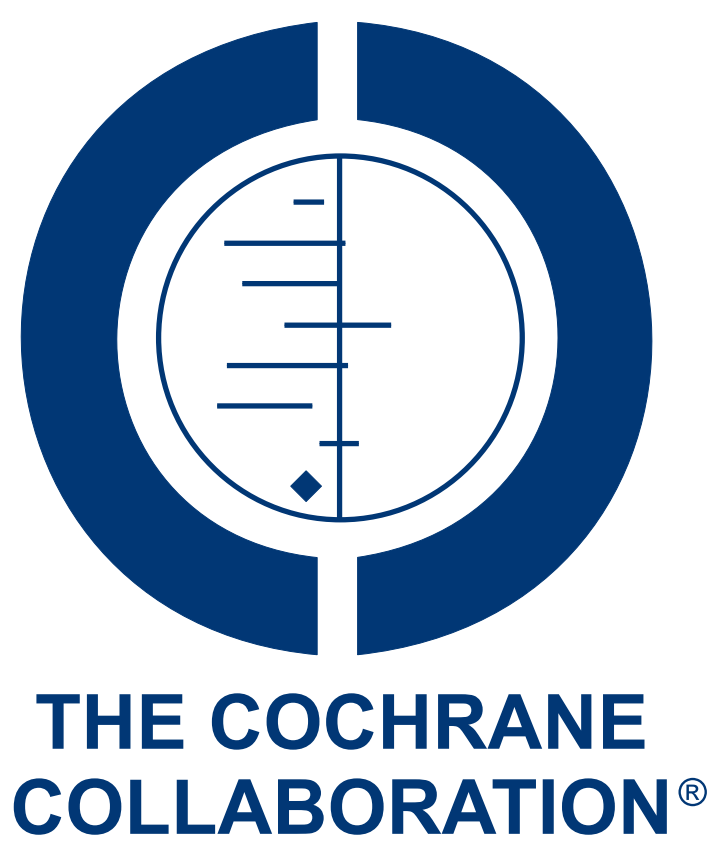

This is a reprint of a Cochrane protocol, prepared and maintained by The Cochrane Collaboration and published in The Cochrane Library 2010, Issue 7

http://www.thecochranelibrary.com

\section{WILEY}

Educational and behavioural interventions for anticoagulant therapy in patients with atrial fibrillation (Protocol)

Copyright @ 2010 The Cochrane Collaboration. Published by John Wiley \& Sons, Ltd. 
TABLE OF CONTENTS

HEADER . . . . . . . . . . . . . . . . . . . . . . . . . . . . . . . . . . . . . . . . 1

ABSTRACT . . . . . . . . . . . . . . . . . . . . . . . . . . . . . . . . . . . . . . . . . . . . 1

BACKGROUND . . . . . . . . . . . . . . . . . . . . . . . . . . . . . . . . . . . . . . . . . . .

OBJECTIVES . . . . . . . . . . . . . . . . . . . . . . . . . . . . . . . . . . . . . . . . . $\quad 2$

METHODS . . . . . . . . . . . . . . . . . . . . . . . . . . . . . . . . . . . . 3

REFERENCES . . . . . . . . . . . . . . . . . . . . . . . . . . . . . . . . . . . . . 6

APPENDICES . . . . . . . . . . . . . . . . . . . . . . . . . . . . . . . . . . . . . 8

HISTORY . . . . . . . . . . . . . . . . . . . . . . . . . . . . . . . . . . . . 10

CONTRIBUTIONS OF AUTHORS . . . . . . . . . . . . . . . . . . . . . . . . . . . . . . . . . . $\quad$.

DECLARATIONS OF INTEREST . . . . . . . . . . . . . . . . . . . . . . . . . . . . . . . . . . . . 11

SOURCES OF SUPPORT . . . . . . . . . . . . . . . . . . . . . . . . . . . . . . . . . . . . . . . . . 11

Educational and behavioural interventions for anticoagulant therapy in patients with atrial fibrillation (Protocol)

Copyright $\odot 2010$ The Cochrane Collaboration. Published by John Wiley \& Sons, Ltd. 
[Intervention Protocol]

\title{
Educational and behavioural interventions for anticoagulant therapy in patients with atrial fibrillation
}

\author{
Danielle E Smith ${ }^{1}$, Christian Borg Xuereb ${ }^{1}$, Helen M Pattison ${ }^{2}$, Gregory YH Lip ${ }^{1}$, Deirdre A Lane ${ }^{1}$ \\ ${ }^{1}$ University of Birmingham Centre for Cardiovascular Sciences, City Hospital, Birmingham, UK. ${ }^{2}$ School of Life and Health Sciences, \\ Aston University, Birmingham, UK
}

Contact address: Deirdre A Lane, University of Birmingham Centre for Cardiovascular Sciences, City Hospital, Dudley Road, Birmingham, B18 7QH, UK. deirdre.lane@swbh.nhs.uk.

Editorial group: Cochrane Heart Group.

Publication status and date: New, published in Issue 7, 2010.

Citation: Smith DE, Borg Xuereb C, Pattison HM, Lip GYH, Lane DA. Educational and behavioural interventions for anticoagulant therapy in patients with atrial fibrillation. Cochrane Database of Systematic Reviews 2010, Issue 7. Art. No.: CD008600. DOI: 10.1002/14651858.CD008600.

Copyright (C) 2010 The Cochrane Collaboration. Published by John Wiley \& Sons, Ltd.

\section{A B S T R A C T}

This is the protocol for a review and there is no abstract. The objectives are as follows:

To assess the effects of educational and behavioural interventions for vitamin $\mathrm{K}$ antagonist anticoagulant therapy in patients with $\mathrm{AF}$.

\section{B A C K G ROU N D}

\section{Description of the condition}

Atrial Fibrillation (AF) is the most common arrhythmia in clinical practice (Fuster 2006) and is associated with a five-fold greater risk of stroke and thromboembolism (Wolf 1991). Prevalence of AF increases with age (Kannel 1998), from 1.5\% at age 50-59 years to $23.5 \%$ at age $80-89$ years (Wolf 1998), as does the incidence of stroke attributable to AF (Lip 2006a). The lifetime risk of developing AF is approximately one in four among people aged 40 years and older (Lloyd-Jones 2007). Assessment of thromboembolic risk determines subsequent treatment plans. There are many stroke risk-stratification models (Stroke Risk in AF Working Group 2007), derived from pooled analysis from the data of antithrombotic treatment trials. The models vary in complexity, but four key clinical features are consistently identified as independent risk factors for stroke: prior stroke or TIA, older age ( $\geq 75$ ), hyper- tension, and diabetes mellitus. The $\mathrm{CHADS}_{2}$ (Gage 2001) stroke risk stratification scheme is simple and well validated scheme, and is the most widely employed. The acronym $\mathrm{CHADS}_{2}$ stands for each of the risk factors: congestive heart failure, hypertension, aged $\geq 75$ years, diabetes mellitus (for which one point is given for each risk factor present), and previous stroke/TIA (which receives two points). Thus the total score ranges from 0 to six. In the United Kingdom, AF patients are risk stratified for stroke using the NICE guidelines (NICE 2006). The NICE guidelines are a practical algorithm based risk stratification. These evidence-based guidelines stratify patients into low, moderate and high risk categories and have similar predictive value for stroke and other vascular events to the $\mathrm{CHADS}_{2}$ scheme (Lip 2006b).

\section{Description of the intervention}

Current guidelines (i.e. Fuster 2006; Gage 2001; Guyatt 2008; NICE 2006) recommend antithrombotic therapy for all patients 
at high risk of stroke $\left(\mathrm{CHADS}_{2} \geq 2\right)$, except for those with contraindications such as peptic ulcers, severe hypertension, bacterial endocarditis, pregnancy, recent surgery (within one month), and a history of intracerebral haemorrhage (NICE 2006). In a metaanalysis, dose-adjusted warfarin (within the International Normalized Ratio (INR) range of 2.0 to 3.0) significantly reduced the risk of ischaemic stroke or embolism in patients with non-valvular AF compared with both aspirin and placebo by $39 \%$ (CI, $22 \%$ to $52 \%$ ) and $64 \%$ (CI, $41 \%$ to $62 \%$ ), respectively (Hart 2007).

Whilst anticoagulant therapy dramatically reduces stroke risk, the therapeutic range of the international normalised ratio (INR) is narrow, and must be maintained. This can be problematic, with INRs $>3.0$ increasing the risk of major and minor bleeding and INRs $<2.0$ increasing the risk of thromboembolism (Lip 2006a). Regular INR monitoring is essential and patients need to carefully adhere to dietary and lifestyle restrictions (Ansell 2004).

A recent retrospective analysis of anticoagulation in the UK demonstrated that only patients with the greatest INR control increased their time to stroke occurrence, with only patients spending $>71 \%$ of their time in target therapeutic range (TTR) benefiting (Morgan 2009). In practice, $51 \%$ of patients at high risk of stroke $\left(\mathrm{CHADS}_{2} \geq 2\right)$ remained outside of their TTR for at least $50 \%$ or more of the time (Morgan 2009). Further, a post hoc analysis of patients enrolled in the Atrial Fibrillation Clopidogrel Trial With Irbesartan for Prevention of Vascular Events (ACTIVE), which randomised AF patients with one additional stroke risk factor to receive clopidogrel $75 \mathrm{mg} / \mathrm{d}$ plus aspirin ( 75 to 100 $\mathrm{mg} / \mathrm{d}$ recommended) or oral anticoagulants (OACs), found that patients with INR values that were within therapeutic range $<58 \%$ of the time gained no benefit from anticoagulation treatment. The INR must be with therapeutic range for $\geq 58 \%$ of the time to confer benefit in terms of stroke risk reduction (Connolly 2008). Thus, maintenance of INR is a major concern for both patients and health care professionals.

Many factors can affect INR control such as non-adherence to prescribed medication (Kumar 1989), inter-drug interactions and variable dietary vitamin $\mathrm{K}$ intake (Holbrook 2005). Poor INR control could also result from the patient's limited knowledge of their condition and their anticoagulant therapy, as several studies have demonstrated poor knowledge of AF and its treatment (Lane 2006; Lip 2002; Nadar 2003; Tang 2003). Studies suggest that knowledge of warfarin therapy correlates significantly with the number of patients with INR values in target range (Tang 2003). In addition, there is an inverse relationship between age and patient knowledge score (Tang 2003), suggesting that patients at highest risk of stroke (aged 80 - 89 years) have the least understanding of their condition and its treatment and are least likely to be within therapeutic INR range (Tang 2003).
The use of OAC requires constant monitoring and thus may be affected by numerous factors, including psychological and practical barriers to adherence. Behavioural and educational interventions targeting patients receiving OACs for various conditions have found a reduced incidence of major bleeding and mortality and increased TTR compared to patients receiving usual care (Beyth 2000; Khan 2004). Beyth 2000 designed a randomised controlled trial for all patients new to warfarin combining INR self monitoring (using a portable monitor to check prothrombin time) and patient education. Throughout six months, the proportion of total treatment time during which the INR was within the therapeutic range was higher in the intervention group than in the usual care group (56\% vs. $32 \%$; $\mathrm{P}<0.001)$. Furthermore, major bleeding was more common in the usual care group than the intervention group (cumulative incidence, $12 \%$ vs. $5.6 \%, \mathrm{P}<0.05$ ). In a similarly designed RCT, Khan 2004 demonstrated that education alone significantly increased TTR during the six months after the intervention began. A brief educational intervention (Lane 2006) specifically targeting AF patients found that whilst there was no significant improvement in patient knowledge of the risks associated with $\mathrm{AF}$, the intervention significantly improved patient knowledge about INR target range and the lifestyle factors that affect INR levels ( $\mathrm{P}=0.001$ and $\mathrm{P}=0.014$ respectively) (Lane 2006). Thus, educational interventions may have an important role to play in optimising health outcomes for AF patients prescribed treatment with warfarin.

\section{Why it is important to do this review}

Patients need sufficient information to make informed choices and actively participate in the management of their own treatment (Thrall 2004). Patient education aims to influence patient behaviour and improve knowledge, attitudes and practices that are necessary to improve health outcomes (Wofford 2008). Techniques used in delivering patient education cover a wide spectrum including the use of booklets and videos as media to transmit additional information, alone or in addition to other self management interventions (such as INR self monitoring) and interventions which used decision aids. Behavioural interventions include interventions that attempt to modify patients' behaviour towards treatment and symptoms such as cognitive behavioural therapy (CBT), motivational interviewing and heart rate variability biofeedback. This review will evaluate the value of educational and behavioural interventions for patients with AF, currently prescribed warfarin. This review will evaluate the impact on the time spent within the therapeutic INR range (TTR) and secondary outcomes such as patient knowledge, quality of life. Psychological well being and illness beliefs will also be evaluated.

\section{How the intervention might work}

\section{O B J E C T I VES}

Educational and behavioural interventions for anticoagulant therapy in patients with atrial fibrillation (Protocol) 
To assess the effects of educational and behavioural interventions for vitamin $\mathrm{K}$ antagonist anticoagulant therapy in patients with $\mathrm{AF}$.

\section{METHODS}

\section{Criteria for considering studies for this review}

\section{Types of studies}

Randomised controlled trials (RCTs) of any type of intervention with any length of follow-up and in any language will be included.

\section{Types of participants}

Adults (aged 18 years or older) with AF (paroxysmal, persistent or permanent), diagnosed and documented by electrocardiogram (12-lead or Holter monitoring), who are eligible for, or currently receiving vitamin $\mathrm{K}$ antagonist anticoagulant therapy will be considered eligible for inclusion in this review. Studies which include AF patients with other medical conditions, will also be included in this review where the studies are RCTs comparing at least one intervention with a control group, and including patients with atrial fibrillation as either the study population or a subgroup. Studies will only be included where patients are grouped per indication i.e. patients taking oral anticoagulants for AF, DVT/PE, valve replacements etc, and only AF patients data will be included within the analysis.

\section{Types of interventions}

All types of educational and behavioural interventions given to $\mathrm{AF}$ patients who were taking vitamin $\mathrm{K}$ antagonist anticoagulation therapy will be considered for this systematic review. Educational interventions include those giving patient information, such as using booklets and videos as media to transmit additional information, alone or in addition to other self management interventions (such as INR self monitoring), interventions which used decision aids, and talking interventions. Behavioural interventions include interventions that attempt to modify patients' behaviour towards treatment and symptoms such as cognitive behavioural therapy $(\mathrm{CBT})$, motivational interviewing and heart-rate variability biofeedback. Interventions could be targeted at adults on the individual level or as a group intervention. The intervention may take place in the emergency department, the hospital, the home or in the community. The intervention could be delivered by a nurse, pharmacist, educator, health or medical practitioner, or a multidisciplinary team associated with the hospital or referred to by the hospital. The intervention could be undertaken at any time point from diagnosis of AF or initiation of vitamin $\mathrm{K}$ antagonist therapy (i.e. not only newly diagnosed AF patients or those newly referred form anticoagulant therapy). Trials will only be considered where the comparison groups are usual care, no intervention, or intervention in combination with other self management techniques. Usual care will be defined as standard anticoagulation clinic practice, where patients attend routine INR checks (defined as usual care by the author). Any length of follow-up will be included.

\section{Types of outcome measures}

\section{Primary outcomes}

The primary outcome measure will be the percentage of time spent within the therapeutic range (TTR), INR (2.0 to 3.0).

\section{Secondary outcomes}

The secondary outcomes will include:

- major bleeding (defined as bleeds that result in death, are life threatening, cause chronic sequelae or consume major health care resources) and minor bleeding (Schulman 2004);

- stroke and thromboembolic events;

- increased knowledge with regards to AFand anticoagulation therapy;

- patient satisfaction;

- acceptability of the anticoagulant therapy;

- quality of life; psychological well being;

- changes in perception towards AF and INR control;

- changes in the patients' illness beliefs and illness representations;

- self reported adherence to treatment and a change in the patients' beliefs about medications;

- economic costs of the intervention (cost-effectiveness).

These outcomes may be quantified using validated or non-validated questionnaires, ratings or scales.

\section{Search methods for identification of studies}

\section{Electronic searches}

We will search the Cochrane Central Register of Controlled Trials (CENTRAL) and the Database of Abstracts of Reviews of Effects (DARE) on The Cochrane Library, MEDLINE, EMBASE, PsycINFO and CINAHL. See Appendix 1 for the search strategies for The Cochrane Library and MEDLINE. These will be adapted for use in the other databases. 


\section{Searching other resources}

Abstract books from national and international cardiology, psychology and psychiatry conferences will be hand-searched. For example:

- Society for Behavioural Medicine and the Division of Health Psychology Conference

- European Health Psychology Conference

- Royal College of Psychiatrists Annual Meeting

Dissertation abstracts (UMI ProQuest Digital Dissertations) will also be searched.

Reference lists of all relevant papers will be searched to identify other potentially relevant articles. We will write to the lead author of all relevant reports and to investigators interested in AF to see if they know of any additional published or unpublished studies that might be relevant to the review.

No language restrictions will be applied to these search strategies.

\section{Data collection and analysis}

\section{Selection of studies}

Two authors (Smith and Borg Xuereb) will scrutinise the titles found from the search and decide on inclusion or exclusion. From the included titles these two authors (Smith and Borg Xuereb) will then select abstracts and papers for inclusion and exclusion. Where disagreements arise on papers to include a third author will make the decision on whether it meets the review criteria (Lane).

\section{Data extraction and management}

Two reviewers will independently extract the data. For each trial, the following data will be extracted using a specially designed data extraction form: participants (sample size, age, sex, ethnicity, marital status, type of Atrial Fibrillation); type of anticoagulation therapy (warfarin, other); type and duration of the interventions (intervention versus usual care or no intervention; other combinations); primary and secondary outcomes (increase in knowledge with regards to AFand anticoagulation therapy; time within the therapeutic INR range; patient satisfaction; acceptability of the anticoagulant therapy; quality of life; changes in perception towards AF and INR control; changes in the patients' illness beliefs and illness representations; change in the patients' beliefs about medications; self reported adherence; psychological well being); length of follow-up; statistical methods employed; the effect size and its precision.

\section{Assessment of risk of bias in included studies}

Two reviewers will independently assess the methodological quality of each trial in accordance with guidelines in the Cochrane
Handbook for Systematic Reviews of Interventions (Higgins 2008). Each study will be assessed on several areas of bias (sequence generation, allocation concealment, degree of blinding, particularly of the outcome assessors, patient attrition rate, selective reporting bias). The risk of bias will be determined using the Collaboration's risk of bias tool.

We will ask if the domains listed below are considered to be adequate. There are three possible responses: Yes, No, or Unclear. Yes indicates a low risk of bias and No indicates a high risk of bias. If insufficient detail is reported the judgement on risk of bias will be Unclear. The criteria for responses is outlined below:

\section{Sequence generation}

Yes, if the allocation sequence was generated using techniques such as a random number table; a computer random number generator; coin tossing; shuffling cards or envelopes; throwing dice.

No, if the allocation sequence was generated using techniques such as odd or even date of birth; date (or day) of admission; hospital or clinic record number.

Unclear, if there was insufficient information about the sequence generation process to permit judgement.

\section{Allocation concealment}

Yes, if the allocation concealment used methods such as central allocation (including telephone, web-based, and pharmacy-controlled randomisation); sequentially numbered drug containers of identical appearance; sequentially numbered opaque, sealed envelopes.

No, if the participants or investigators enrolling participants could possibly foresee assignments and thus introduce selection bias such as allocation based on using an open random allocation schedule (e.g. a list of random numbers); assignment envelopes used without appropriate safeguards (e.g. if envelopes were unsealed or nonopaque, or not sequentially numbered); alternation or rotation; date of birth; case record number.

Unclear, if there was insufficient information to permit judgement of Yes or No. If the method of concealment was not described or not described in sufficient detail to allow a definite judgement (e.g. if the use of assignment envelopes was described but it remained unclear whether envelopes were sequentially numbered, opaque and sealed).

Where the method of allocation is unclear, we plan to contact study authors to provide further details.

Blinding 
Yes, if there was no blinding but the review authors judged that the outcome and the outcome measurement was not likely to be influenced by lack of blinding; if blinding of participants and key study personnel was ensured and it was unlikely that the blinding could have been broken; if either participants or some key study personnel were not blinded but outcome assessment was blinded and the non-blinding of others was unlikely to introduce bias.

No, if there was no blinding or incomplete blinding and the outcome or outcome measurement was likely to be influenced by lack of blinding; if blinding of key study participants and personnel was attempted but it was likely that the blinding could have been broken; if either participants or some key study personnel were not blinded and the non-blinding of others was likely to introduce bias.

Unclear, if there was insufficient information to permit judgement of Yes or No or the study did not address this outcome (e.g. where the blinding was described only as double-blind without any other details).

\section{Incomplete data assessment (loss of participants, for example with withdrawals, dropouts, protocol deviations)}

Yes, if there were no missing outcome data; reasons for missing outcome data were unlikely to be related to the true outcome; missing outcome data were balanced in numbers across intervention groups with similar reasons for missing data across groups; for dichotomous outcome data, the proportion of missing outcomes compared with observed event risk was not enough to have a clinically relevant impact on the intervention effect estimate; for continuous outcome data, plausible effect size (difference in means or standardised difference in means) among missing outcomes was not enough to have a clinically relevant impact on observed effect size; missing data were imputed using appropriate methods.

No, if the reasons for missing outcome data were likely to be related to true outcome, with either imbalance in numbers or reasons for missing data across intervention groups; for dichotomous outcome data, the proportion of missing outcomes compared with observed event risk was enough to introduce clinically relevant bias in the intervention effect estimate; for continuous outcome data, plausible effect size (difference in means or standardised difference in means) among missing outcomes was enough to introduce clinically relevant bias in observed effect size; 'as-treated' analysis done with substantial departure of the intervention received from that assigned at randomisation; potentially inappropriate application of simple imputation.

Unclear, if there was insufficient reporting of attrition or exclusions to permit judgement of Yes or No (e.g. numbers randomised were not stated, no reasons for missing data were provided); or the study did not address this.

\section{Selective outcome reporting}

Yes, if the study protocol was available and all of the study's prespecified (primary and secondary) outcomes that were of interest in the review were reported in the pre-specified way; the study protocol was not available but it was clear that the published reports included all expected outcomes, including those that were pre-specified.

No, if not all of the study's pre-specified primary outcomes were reported; one or more primary outcomes were reported using measurements, analysis methods or subsets of the data (e.g. subscales) that were not pre-specified; one or more reported primary outcomes were not pre-specified (unless clear justification for their reporting was provided, such as an unexpected adverse effect); one or more outcomes of interest in the review were reported incompletely so that they could not be entered in a meta-analysis; the study report failed to include results for a key outcome that would be expected to have been reported for such a study.

Unclear, if there was insufficient information to permit judgement of Yes or No.

\section{Other sources of bias}

Yes, if the study appeared to be free of other sources of bias.

No, if there was at least one important risk of bias (e.g. the study had a potential source of bias related to the specific study design used; stopped early due to some data-dependent process (including a formal-stopping rule); had extreme baseline imbalance; had been claimed to be fraudulent; had some other problem).

Unclear, if there was either insufficient information to assess whether an important risk of bias existed or if there was insufficient rationale or evidence that an identified problem would introduce bias.

\section{Measures of treatment effect}

Statistical analysis would be undertaken as follows: for continuous variables (e.g. changes in illness perception questionnaire or changes in time spent within INR therapeutic range), the weighted mean difference would be used. As a summary measure of effectiveness, odds ratios with $95 \%$ confidence intervals will be calculated for dichotomous variables and where possible, the number needed to treat (NNT) statistic would also be calculated.

\section{Dealing with missing data}


We will try to contact the authors for studies with incomplete information in published articles and for data clarifications if required. Investigators of unpublished trials will also be contacted.

\section{Assessment of heterogeneity}

Where relevant RCTs are identified assessment of their heterogeneity will be carried out. The $\mathrm{I}^{2}$ statistic will be examined to describe the proportion of the variability in the results that is due to heterogeneity. In addition, Chi-squared test for heterogeneity will be performed and data will be considered heterogenous if $\mathrm{P}<$ 0.10 . If significant heterogeneity is detected, possible explanations will be looked for such as differences in population, the intervention, setting and outcomes assessed.

\section{Assessment of reporting biases}

Publication bias will be tested by funnel plot based on the data for the primary outcome of time spent within therapeutic INR range. Asymmetry of the funnel plot will be taken as an indication of publication bias. Other causes of asymmetry of funnel plot will also be looked at like clinical heterogeneity between studies (for example different control event rates) or methodological heterogeneity between studies (for example failure to conceal allocation). A summary of who was blinded during both the conduct and analysis of the study will also be summarised and conclusions will inform the risk-of-bias tool. The completeness of the data will be summarised and any concerns over the exclusion of participants and/or excessive drop-out will be reported. Concerns over the selective reporting of outcomes, time-points or sub-groups will also be reported.

\section{Data synthesis}

Results of individual studies will initially be combined within a narrative review. This will take into account methodological quality of the study. Where possible and appropriate, meta-analysis will be used to statistically combine results. We will calculate the percentage change (i.e. the per cent improvement relative to the post intervention average in the control group). If insufficient data are present to conduct a meta-analysis, we will report effect sizes and confidence intervals (CIs) of the included studies using a standard method of presentation. TTR data will only be included if directly reported, where available from personal communication with the authors, or if all INR results for each participant for the duration of the trial are available to permit calculation of TTR using the Rosendaal method (Rosendaal 1993).

\section{Subgroup analysis and investigation of heterogeneity}

Subgroup analyses will be carried out looking at the type of intervention (educational alone, behavioural alone, and a combination of education and behavioural vs. usual care), frequency (one session vs. multiple sessions) and duration ( $<6$ months / $>6$ months) of the intervention, length of time on OAC, men vs. women, individual vs. group interventions and age of participant groups, dependant upon the availability of such data in the included study reports.

\section{Sensitivity analysis}

Sensitivity analyses would be employed to examine factors that may lead to differences between the results of individual trials: poor quality versus good quality trials.

\section{R E F E R E N C E S}

\section{Additional references}

\section{Ansell 2004}

Ansell J, Hirsh J, Poller L, Bussey H, Jacobsen A, Hylek

E. The pharmacology and management of the vitamin $\mathrm{K}$ antagonists. Chest 2004;126:204-33.

\section{Beyth 2000}

Beyth RJ, Quin L, Landefeld S. A multicomponent intervention to prevent major bleeding complications in older patients receiving warfarin. Annals of Internal Medicine 2000;133:687-95.

\section{Connolly 2008}

Connolly SJ, Pogue J, Eikelboom J, Flaker G, Commerford P, Franzosi MG, et al.Benefit of oral anticoagulant over antiplatelet therapy in atrial fibrillation depends on the quality of international normalised ratio control achieved by centres and countries as measured by time in therapeutic range. Circulation 2008;118:2029-37.
Fuster 2006

Fuster V, Rydén LE, Cannom DS, Crijns HJ, Curtis $A B$, Ellenbogen KA, et al.ACC/AHA/ESC 2006 guidelines for the management of patients with atrial fibrillation: a report of the American College of Cardiology/American Heart Association Task Force on Practice Guidelines and the European Society of Cardiology Committee for Practice Guidelines (Writing Committee to Revise the 2001 Guidelines for the Management of Patients With Atrial Fibrillation). Circulation 2006;114:257-354.

Gage 2001

Gage BF, Waterman AD, Shannon W, Boechler M, Rich MW, Radford MJ. Validation of clinical classification schemes for predicting stroke: results from the National Registry of Atrial Fibrillation. JAMA 2001;285:2864-70.

Guyatt 2008

Guyatt GH, Cook DJ, Jaeschke R, Pauker SG, Schunemann HJ. Grades of recommendation for antithrombotic agents. 
CHEST 2008;133(6 suppl):123S-31S.

\section{Hart 2007}

Hart RG, Pearce LA, Aguilar MI. Meta-analysis: antithrombotic therapy to prevent stroke in patients who have non-valvular atrial fibrillation. Annals of Internal Medicine 2007;146:857-67.

Higgins 2008

Higgins JPT, Green S (editors). Cochrane Handbook for Systematic Reviews of Interventions Version 5.0.1. The Cochrane Collaboration, 2008.

\section{Holbrook 2005}

Holbrook AM, Pereira JA, Labiris R, McDonald H, Douketis JD, Crowther M. Systematic overview of warfarin and its drug and food interactions. Archives of Internal Medicine 2005;165:1095-1106.

\section{Kannel 1998}

Kannel WB, Wolf PA, Benjamin EJ, Levy D. Prevalence, incidence, prognosis, and predisposing conditions for atrial fibrillation: population-based estimates. American Journal of Cardiology 1998;82(8A):2-9.

\section{Khan 2004}

Khan TI, Kamali F, Kesteven P, Avery P, Wynne H. The value of education and self monitoring in the management of warfarin therapy in older patients with unstable control of anticoagulation. British Journal of Haematology 2004; 126:557-64.

\section{Kumar 1989}

Kumar S, Haigh JR, Rhodes LE, Peaker S, Davies JA, Roberts $\mathrm{BE}$, et al.Poor compliance is a major factor in unstable outpatient control of anticoagulant therapy. Journal of Thrombosis and Haemostasis 1989;62:729-32.

Lane 2006

Lane DA, Ponsford J, Shelley A, Sirpal A, Lip GYH. Patient knowledge and perceptions of atrial fibrillation ans anticoagulant therapy. Journal of Thrombosis and Haemostasis 2006;110:354-8.

Lip 2002

Lip GYH, Kamath S, Jafri M, Mohammed A, Bareford D, McAlister FA. Ethnic differences in patient perceptions of atrial fibrillation and anticoagulation therapy: the West Birmingham Atrial Fibrillation Project. Stroke 2002;33: $238-44$.

Lip 2006a

Lip GY, Edwards SJ. Stroke prevention with aspirin, warfarin and ximelagatran in patients with non-valvular atrial fibrillation: a systematic review and meta-analysis. Thrombosis Research 2006;118:321-33.

Lip 2006b

Lip GYH, Lane D, van Walraven C, Hart R. The additive role of plasma von Willebrand Factor levels to clinical factors for risk stratification in patients with atrial fibrillation. Stoke 2006;37:2294-300.

Lloyd-Jones 2007

Lloyd-Jones DM, Wang TJ, Leip EP, Larson MG, Levy D, Vasan RS, et al.Lifetime risk for developing atrial fibrillation: The Framingham Heart Study. Circulation 2007;110:1042-6.

Morgan 2009

Morgan CL, McEwan P, Tukiendorf A, Robinson PA, Clemens A, Plumb JM. Warfarin treatment in patients with atrial fibrillation: Observing outcomes associated with varying levels of INR control. Thrombosis 2009;124:37-41.

Nadar 2003

Nadar S, Begum N, Kaur B, Sandhu S, Lip GYH. Patients understanding of anticoagulation therapy in a multiethnic population. Journal of the Royal Society of Medicine 2003; 96:175-9.

\section{NICE 2006}

National Collaborating Centre for Chronic Conditions. Atrial fibrillation: national clinical guideline for management in primary and secondary care. London: Royal College of Physicians 2006.

\section{Rosendaal 1993}

Rosendaal FR, Cannegieter SC, van der Meer FJM, Briet E. A method to determine the optimal intensity of oral anticoagulant therapy. Thrombosis and Haemostasis 1993;69 (3):236-9.

Schulman 2004

Schulman S, Kearon C. Definition of major bleeding in clinical investigations of antihemostatic medicinal products in non-surgical patients. Journal of Thrombosis and Haemostasis 2005;3:692-4

Smith 2010

Smith DE, Borg Xuereb C, Pattison HM, Lip GYH, Lane DA. TRial of an Educational intervention on patients' knowledge of Atrial fibrillation and anticoagulant therapy, INR control and outcome of Treatment with warfarin (TREAT). BMC Cardiovascular Disorders 2010;10(21).

Stroke Risk in AF Working Group 2007 Stroke Risk in Atrial Fibrillation Working Group. Independent predictors of stroke in patients with atrial fibrillation: a systematic review. Neurology 2007;132:8-10.

Tang 2003

Tang E, Lai C, Lee K, Wong RSM, Cheng G, Chan TYK. Relationship between patients warfarin knowledge and anticoagulation control. Annals of Pharmacotherapy 2003; 37:34-9.

Thrall 2004

Thrall G, Lip GYH, Lane D. Compliance with pharmacological therapy in hypertension: can we do better, and how?. Journal of Human Hypertension 2004;18:595-7.

\section{Wofford 2008}

Wofford JL, Wells MD, Singh S. Best strategies for patient education about anticoagulation with warfarin: a systematic review. BMC Health Service Research 2008;8(40).

\section{Wolf 1991}

Wolf PA, Abbott RD, Kannel WB. Atrial fibrillation as an independent risk factor for stroke: the Framingham Study. Stroke 1991;22(8):983-8. 


\section{Wolf 1998}

Wolf PA, Mitchell JB, Baker CS, Kannel WB, D’Agostino

RB. Impact of atrial fibrillation on mortality, stroke, and

medical costs. Archives Internal Medicine 1998;158(3):

229-34.

* Indicates the major publication for the study

\section{A P P E N D I C E S}

\section{Appendix I. Search strategy}

\section{The Cochrane Library}

\#1 MeSH descriptor patient education as topic this term only \#2 MeSH descriptor attitude to health explode all trees \#3 MeSH descriptor patient participation this term only \#4 MeSH descriptor behavior therapy this term only \#5 MeSH descriptor cognitive therapy this term only \#6 MeSH descriptor counseling explode all trees \#7 MeSH descriptor motivation this term only \#8 MeSH descriptor goals this term only \#9 MeSH descriptor Biofeedback (Psychology) this term only \#10 MeSH descriptor decision support techniques this term only \#11 MeSH descriptor Communications Media explode all trees \#12 education in All Text

\#13 (training in All Text or train in All Text)

\#14 (teaching in All Text or teach in All Text)

\#15 (behaviour* in All Text or behavior* in All Text)

\#16 "patient knowledge" in All Text

\#17 counsel* in All Text

\#18 (cognitiv* in All Text near/3 therapy in All Text)

\#19 (cognitiv* in All Text near/3 intervention* in All Text)

\#20 motivation* in All Text

\#21 contingency next management in All Text

\#22 (biofeedback in All Text or bio-feedback in All Text)

\#23 (goal in All Text or goals in All Text)

\#24 (decision* in All Text near/3 aid* in All Text)

\#25 pamphlet* in All Text

\#26 booklet* in All Text

\#27 video* in All Text

\#28 decision next aid* in All Text

\#29 "patient participation" in All Text

$\# 30$ (\#1 or \#2 or \#3 or \#4 or \#5 or \#6 or \#7 or \#8 or \#9 or \#10)

$\# 31$ (\#11 or \#12 or \#13 or \#14 or \#15 or \#16 or \#17 or \#18 or \#19 or \#20)

$\# 32$ (\#21 or \#22 or \#23 or \#24 or \#25 or \#26 or \#27 or \#28 or \#29)

$\# 33$ (\#30 or \#31 or \#32)

\#34 MeSH descriptor warfarin this term only \#35 MeSH descriptor Coumarins explode all trees

Educational and behavioural interventions for anticoagulant therapy in patients with atrial fibrillation (Protocol)

Copyright ( 2010 The Cochrane Collaboration. Published by John Wiley \& Sons, Ltd. 
\#36 MeSH descriptor anticoagulants this term only

\#37 MeSH descriptor vitamin k explode all trees with qualifiers: AI

\#38 oral next anticoagula* in All Text

\#39 Oral next anti-coagula* in All Text

\#40 ("vitamin K" in All Text and (antagonist* in All Text or inhibitor* in All Text))

\#41 "antivitamin K" in All Text

\#42 "anti-vitamin K" in All Text

\#43 warfarin in All Text

\#44 acenocoumarol in All Text

\#45 sintrom in All Text

\#46 sinthrome in All Text

\#47 jantoven in All Text

\#48 marevan in All Text

\#49 coumadin* in All Text

\#50 waran in All Text

\#51 phenprocoumon in All Text

\#52 nicoumalone in All Text

\#53 VKA in All Text

\#54 coumarin* in All Text

\#55 dicoumarol in All Text

\#56 dicumarol in All Text

$\# 57$ (\#34 or \#35 or \#36 or \#37 or \#38 or \#39 or \#40 or \#41 or \#42 or \#43)

$\# 58$ (\#44 or \#45 or \#46 or \#47 or \#48 or \#49 or \#50 or \#51 or \#52 or \#53 or \#54 or \#55 or \#56)

\#59 (\#57 or \#58)

\#60 (\#33 and \#59)

\section{MEDLINE on Ovid}

1. Warfarin/

2. acenocoumarol/

3. Coumarins/

4. Phenindione/

5. Dicumarol/

6. Anticoagulants/

7. oral anticoagula $\$ . t w$.

8. exp Vitamin K/ai [Antagonists \& Inhibitors]

9. warfarin.tw.

10. acenocoumarol.tw.

11. sintrom.tw.

12. sinthrome.tw.

13. jantoven.tw.

14. marevan.tw.

15. coumadin\$.tw.

16. waran.tw.

17. Phenprocoumon/

18. nicoumalone.tw.

19. (vitamin $\mathrm{k}$ adj3 antagonist\$).tw.

20. vitamin $\mathrm{k}$ inhibitor\$.tw.

21. oral anticoagula $\$$. tw.

22 . oral anti-coagula $\$ . t w$.

23. vka.tw.

24. antivitamin k.tw.

25. anti-vitamin k.tw.

Educational and behavioural interventions for anticoagulant therapy in patients with atrial fibrillation (Protocol)

Copyright ( 2010 The Cochrane Collaboration. Published by John Wiley \& Sons, Ltd. 
26. or/1-25

27. Patient Education as Topic/

28. exp Attitude to Health/

29. Patient Participation/

30. ((educat\$ or train $\$$ or teach\$) adj3 (program\$ or intervention\$)).tw.

31. (patient $\$$ adj3 (train $\$$ or teach $\$$ or educat $\$$ or inform $\$$ )).tw.

32. patient knowledge.tw.

33. Behavior Therapy/

34. Cognitive Therapy/

35. exp counseling/

36. (behavi $\$$ adj3 (therap\$ or manage $\$$ or modif $\$$ or chang $\$$ or intervention $\$$ )).tw.

37. (cogniti adj3 (therap\$ or intervention\$)).tw.

38. counsel\$.tw.

39. Motivation/

40. motivational interview\$.tw.

41. contingency management.tw.

42. biofeedback.tw.

43. bio-feedback.tw.

44. goals/

45. (goal\$ adj3 set $\$)$.tw.

46. decision support techniques/

47. decision\$ aid\$.tw.

48. exp communications media/

49. pamphlet\$.tw.

50. booklet $\$$. tw.

51. video\$.tw.

52. or/27-51

53. 26 and 52

54. randomized controlled trial.pt.

55. controlled clinical trial.pt.

56. randomized.ab.

57. placebo.ab.

58. clinical trials as topic.sh.

59. randomly.ab.

60. trial.ti.

61.54 or 55 or 56 or 57 or 58 or 59 or 60

62. exp animals/ not humans.sh.

63. 61 not 62

64.53 and 63

\section{H I S T O R Y}

Protocol first published: Issue 7, 2010 


\section{CONTRIBUTIONSOFAUTHORS}

Data collection, paper searches, screening and appraisal as well as data extraction will all be carried out by Smith and Borg Xuereb. Dr Lane will advise on extraction method, analysis and general review processes. Interpretation of analysis and final drafts of the review will be overseen by all of the authors.

\section{DECLARATIONSOF INTEREST}

DES is carrying out a PhD studentship that is funded by an Investigator-Initiated Educational Grant from Bayer Healthcare and Aston University. CBX has no conflict of interest to declare. HMP is currently funded by the National Institute of Health Research, ESRC and Breast Cancer Campaign. She has no conflicts of interest arising from current or past activities. GYHL has no conflicts of interest to declare. DAL is the principal grant holder for TREAT. DES is currently the primary investigator for the 'TRial of an Educational intervention on patients' knowledge of Atrial fibrillation and anticoagulant therapy, INR control, and outcome of Treatment with warfarin' (TREAT). The trial protocol manuscript has been accepted for publication within BMC Cardiovascular Disorders Smith 2010. The TREAT results will not be available until 2012. DAL, GHYL and HP are supervising this study. This review is not funded by Bayer healthcare.

\section{SOURCES OF SUPPORT}

\section{Internal sources}

- No sources of support supplied

\section{External sources}

- New Source of support, Not specified.

This review is funded by University of Birmingham Centre for Cardiovascular Sciences, City Hospital and Aston University. Bayer Healthcare are not funding this review, but are providing support to DS who is one author of the review. 